\title{
Estudio de una salida urbana para el aprendizaje de la geología de bachillerato
}

\author{
Nahia Seijas-Garzón ${ }^{1}$, Maite Morentin-Pascual ${ }^{2}$ \\ ${ }^{1}$ Estudiante de doctorado en Psicodidáctica, Campus de Leioa, Universidad del País Vasco UPV/EHU. \\ nseijas001@ikasle.ebu.eus \\ ${ }^{2}$ Departamento de Didáctica de la Matemática y de las Ciencias Experimentales, Campus de Leioa, \\ Universidad del Pais Vasco UPV/EHU. maite.morentin@ehu.eus
}

[Recibido: 22 junio 2017. Revisado: 29 diciembre 2017. Aceptado: 25 enero 2018]

\begin{abstract}
Resumen: En este estudio se presenta una propuesta didáctica para la enseñanza de la geología llevada a cabo con un grupo de estudiantes de $1^{\circ}$ de Bachillerato: una salida urbana como recurso para trabajar las rocas y los procesos petrogenéticos. Con el conjunto de actividades que envuelven a la salida (anteriores, durante y posteriores a ella) se pretende que el alumnado adquiera competencias y contenidos del currículo y para ello se ha trabajado mediante la metodología de resolución de problemas. La intervención se ha realizado en Orduña (Bizkaia) y, en este trabajo, además de describir la secuencia didáctica utilizada, se analizan los resultados obtenidos mediante diferentes instrumentos de evaluación, y se plantean varias conclusiones que pueden servir para su utilización futura por el profesorado.
\end{abstract}

Palabras clave: didáctica de la geología, propuesta didáctica, salida urbana, evaluación.

Educational study of an urban trip for the learning of geology at highschool

Abtsract: This paper presents an educational proposal for the teaching of geology developed for a group of high school students: a urban trip as a resource to learn about rocks and petrogenetic processes. The aim of the activities related to the field trip (activities made before, during, and after the outing) is that the students achieve the competences and contents on the curriculum, and, in order to accomplish that goal, a method of problem solving has been used. The intervention has taken place in Orduña (Biscay), with pupils from the school Ntra. Sra. de la Antigua located in that city; and this paper includes the description of the didactic sequence used and the discussion about the results obtained by different assessment tools.

Keywords: didactics of geology, educational proposal, urban trips, evaluation.

Para citar este artículo: Seijas N., Morentin M. (2018) Estudio de una salida urbana para el aprendizaje de la geología en Bachillerato. Revista Eureka sobre Enseñanza y Divulgación de las Ciencias 15 (2), 2107. doi: 10.25267/Rev_Eureka_ensen_divulg_cienc.2018.v15.i2.2107

\section{Introducción}

Vivimos en un planeta en el que suceden constantemente procesos geológicos de distinta magnitud y duración, internos y externos, que inevitablemente nos afectan y pueden repercutir en nuestro día a día. Parece lógico afirmar, por tanto, que comprender el funcionamiento de nuestro planeta a diferentes escalas debe ser un objetivo básico de la Educación Secundaria.

Sin embargo, según Pedrinaci (2012a), la geología escolar actual se aleja notablemente de lo deseable. Los problemas a los que se enfrenta esta ciencia en el ámbito educativo se resumen en tres aspectos: que la formación científica en sí misma recibe, en general, poca atención durante la etapa de Educación Secundaria; que la geología es una de las ciencias más desconocidas en la sociedad y menos representadas en el currículo; y, por último, que los contenidos geológicos que se trabajan en el aula suelen ser más escasos que los presentes en los currículos oficiales. 
Para hacer frente a esta situación, diferentes autores han propuesto (Brusi et al. 2011, Cañas y Martín Díaz 2010, entre otros) nuevas formas de enseñar ciencia y, más concretamente, geología; éstas atienden a la nueva perspectiva competencial de los currículos oficiales, a la necesidad de trabajar contenidos de tipo procedimental y actitudinal además de los conceptuales, a la relación de la ciencia con la tecnología y la sociedad, etc. A este respecto la aportación probablemente más interesante es el documento "Alfabetización en ciencias de la Tierra" (Pedrinaci et al. 2012), documento que constituye un manifiesto de un nuevo modo de enseñar y aprender geología.

Esta nueva visión sobre la enseñanza de las ciencias de la Tierra requiere de nuevas metodologías, que se alejen de la mera transmisión de conceptos y de la geología fuera de contexto. A este efecto, el trabajo que presentamos quiere subrayar el valor didáctico de las salidas de campo, siempre que se realicen de manera adecuada, para "alfabetizar" en ciencias de la Tierra. Se han publicado ya varios trabajos que demuestran que una salida de campo correctamente organizada posibilita un aprendizaje significativo de la geología (Pedrinaci, Sequeiros y García de la Torre 1994, Del Toro y Morcillo 2011, Zamalloa et al. 2014), y la intervención que proponemos pretende reforzar esta afirmación.

En concreto, en este trabajo se presenta una experiencia desarrollada en el Colegio Ntra. Sra. de la Antigua de Orduña (Bizkaia), con un grupo de $1^{\circ}$ de Bachillerato, cuyo núcleo fundamental fue la realización de una salida urbana para analizar las rocas de algunos edificios.

\section{Justificación teórica}

\section{¿Por qué realizar salidas de campo?}

Tal y como señala la bibliografía, las salidas de campo pueden ser beneficiosas en dos ámbitos principalmente: en la motivación del alumnado y en el aprendizaje de contenidos.

La motivación tiene una importancia especial porque es reconocida como un factor fundamental para que ocurra el aprendizaje significativo. El tipo de motivación más beneficiosa para el proceso enseñanza-aprendizaje es la definida por Ryan y Deci (2000) como: "motivación intrínseca al aprendizaje". Esta se da cuando el alumnado consigue estar concentrado en el trabajo, sin aburrirse y sin sentir ansiedad; cuando se busca información y/o se pregunta para resolver dudas; y, en definitiva, cuando se es capaz de auto-regular el proceso de aprendizaje. Así, las salidas de campo parecen un contexto idóneo para fomentar este tipo de motivación, ya que el entorno se presta a que los estudiantes aprendan de forma autónoma y activa y desarrollen actitudes positivas hacia la ciencia; como algunos autores reconocen (Cañal 2012) dichas actitudes son un factor fundamental en el desarrollo de la competencia científica y viceversa.

En referencia al ámbito del aprendizaje de contenidos, varios autores (Pedrinaci et al. 1994, Del Toro y Morcillo 2011) han subrayado que las salidas de campo son muy útiles para el aprendizaje de conceptos (por ej. entender lo que son los sedimentos, los estratos, las fallas, etc.), procedimientos (observación, toma de datos, uso de mapas, etc.) y actitudes (trabajar correctamente en grupo, respeto y sostenibilidad hacia el entorno, etc.); en resumen, son actividades que potencian el desarrollo de la competencia científica partiendo de un enfoque centrado en el contexto. Aunque la concepción de competencia es un concepto en construcción, el último informe PISA (OECD 2016) ya discrimina entre el conocimiento de estrategias para "hacer ciencia" del conocimiento sobre "qué entendemos por ciencia", añadiendo el campo de las actitudes (Sanmartí y Márquez 2017). 
Sin embargo, según Pedrinaci (2012b), existen ventajas aún más importantes que convierten las salidas de campo en actividades imprescindibles para el aprendizaje significativo de la geología. En primer lugar, salir al campo reduce la distancia de abstracción de contenidos conceptuales y procedimentales (por ejemplo, la distancia de abstracción entre una falla representada en un libro de texto y una en la realidad solo puede ser salvada con una observación directa). Por otro lado, obliga a establecer relaciones entre los diferentes apartados de la geología, para poder resolver satisfactoriamente un problema real. Por último, este tipo de trabajo ayuda a superar la falta de seguridad del alumnado, pues se acostumbra a resolver con paciencia y mucho análisis los problemas abiertos que se presentan en el campo.

En concreto, si nos ceñimos a las salidas urbanas, diversos autores han manifestado el potencial de las ciudades como recursos educativos para el aprendizaje de la geología (Corbí et al. 2013, Fuertes et al. 2016), señalando la amplia variedad de rocas ornamentales que se pueden observar, la facilidad con la que se puede acceder a ellas y las conexiones que permiten hacer con el patrimonio geológico e histórico. Aunque no sea un escenario perfecto (no se ven las rocas en su contexto geológico) constituye un valioso recurso para trabajar diversos aspectos del currículo de Bachillerato, como se verá en este artículo.

\section{El diseño de las salidas}

Para garantizar el aprendizaje significativo basado en el modelo constructivista de aprendizaje, las salidas del aula tienen que estar relacionadas con el currículo e integradas en la planificación escolar, según el marco teórico "Visitas Centradas en el Aprendizaje” propuesto por Morentin y Guisasola (2013). Las ideas recogidas en él se resumen en tres principios:

1- Establecer conexiones entre el contexto del aula y el de la salida, integrando ésta en la planificación escolar y estableciendo objetivos concretos y criterios de evaluación.

2- Estructurar adecuadamente las actividades relacionadas con la salida, realizando actividades previas, durante y posteriores a la salida.

3- Trabajar con nuevas estrategias metodológicas. Se proponen, entre otras: implicar a los estudiantes activamente, realizar trabajos en grupo enfatizando la comunicación escrita y verbal, y fomentar el interés y la motivación intrínseca.

En consonancia con este marco teórico hemos adoptado el método de aprendizaje basado en problemas, consistente en plantear un problema de la vida real frente al que el estudiantado identifique las necesidades de aprendizaje y busque información necesaria para su resolución (Branda 2009). Esta metodología favorece mejoras en varias dimensiones de la competencia científica, como son la emisión de hipótesis, la planificación de la experimentación, etc. (Pavón y Martínez Aznar 2014) al tiempo que enfrenta a los estudiantes a situaciones abiertas que deben reformular y definir. Con este fundamento y en relación a las salidas de campo en la asignatura de geología se sitúa el tipo de salida "como tratamiento de problemas de investigación" propuesto por Pedrinaci y colaboradores (1994), en la que se hace al alumnado protagonista de su aprendizaje también fuera del aula.

Lo que se plantea es comenzar formulando un problema antes de la salida, que esté bien contextualizado y relacionado con el currículo, y al que se le pueda hacer frente desde diversas perspectivas teóricas. Antes de salir del aula se les pide a los estudiantes que trabajen de forma cooperativa para realizar una guía, de la que se valgan en la salida para resolver el problema planteado. Este proceso tiene una importancia fundamental, ya que de él depende el aprovechamiento de la salida. Por último, durante la salida y después de terminar ésta, los grupos de trabajo deberán llegar a unas conclusiones y compartirlas con sus compañeros y compañeras. 
En cuanto a la evaluación, se utilizarán diferentes herramientas en función de la actividad y del tipo de contenido a evaluar, ya que coincidimos con Sanmartí (2007) en que dicha evaluación debe ser sobre todo reguladora del proceso de aprendizaje; sin embargo, somos conscientes que la salida del aula influirá especialmente en las actitudes de los estudiantes, pero la construcción de conocimiento estará relacionada también con el trabajo paralelo a desarrollar en el aula (Vilaseca y Bach, 1993).

\section{Metodología}

La experiencia que presentamos a continuación consta de 3 apartados principales: el diseño de la secuencia didáctica de la salida urbana, la puesta en práctica de la misma y, su posterior valoración junto con las implicaciones didácticas correspondientes.

Esta secuencia está encuadrada en una unidad didáctica titulada "Las rocas y sus orígenes", en la que se trabajan de manera integradora los tipos de rocas y los procesos formadores de estas. Este tema es el primer contacto que el grupo de estudiantes tiene con la geología en $1^{\circ}$ de Bachillerato según la programación anual del centro.

\section{Objetivos y contexto del estudio}

El grupo con el que se ha trabajado es la clase de $1^{\circ}$ de Bachillerato Científico del Colegio Ntra. Sra. De la Antigua de Orduña (Bizkaia), en la asignatura Biología y Geología. Está compuesto por 13 alumnos y alumnas, que se dividieron en tres grupos de tres estudiantes y uno de cuatro para la realización de la experiencia.

Este trabajo tiene como objetivo principal desarrollar la competencia científica del alumnado, entendida ésta como la integración de una serie de capacidades para utilizar el conocimiento científico en contextos concretos, para comprender los rasgos característicos de la ciencia, para formular e investigar problemas e hipótesis, etc.

Para conseguir este objetivo, se diseñó una unidad didáctica en la que se trabajan varios contenidos del currículo de Bachillerato del País Vasco (Decreto 127/2016), y su valoración posterior nos indicará el grado de consecución de dicho objetivo.

Los contenidos conceptuales elegidos son "los procesos petrogenéticos y las rocas magmáticas, metamórficas y sedimentarias más representativas", incluidos en los bloques $7^{\circ} \mathrm{y}$ $9^{\circ}$ del citado currículo oficial. Además, se trabajarán contenidos procedimentales y actitudinales de dicho currículo (incluidos en el $1^{\circ}$ bloque de contenidos); procedimientos como la formulación de hipótesis, identificación y control de variables, diseño experimental, interpretación crítica de los resultados y comunicación de estos, técnicas para el trabajo de campo, elaboración de informes...; y actitudes como el trabajo cooperativo, la exactitud en la utilización del lenguaje científico, el esfuerzo y la responsabilidad en las tareas de grupo y en el trabajo personal, la motivación intrínseca hacia la materia, y, especialmente, el interés mostrado por la geología.

Para poder trabajar todos estos contenidos de una manera significativa, es interesante tener en cuenta cuáles son las ideas previas típicas de este tema, obstáculos que los alumnos deberán identificar y superar (Pedrinaci 1996, Ramos et al. 2001):

- No entienden las rocas como una mezcla heterogénea; desconocen que están formadas por minerales y piensan que los minerales son elementos raros que a veces se encuentran en la naturaleza.

- Piensan que los fósiles que contienen las rocas son más antiguos que éstas. 
- Tienen una visión estática de la Tierra: ven el relieve como un elemento muy estable, y explican los cambios ocurridos desde un punto de vista catastrofista (terremotos); o, en cualquier caso, siempre mediante procesos destructivos (erosión).

- No entienden que los procesos constructivos ocurren por acumulación en vez de por interacción, y sólo consideran el volcanismo.

Teniendo en cuenta los objetivos mencionados y las ideas alternativas esperables, se diseñó e implementó la unidad didáctica "Las rocas y sus orígenes" dividida en 5 apartados, incluyendo la salida urbana en el último:

1. Rocas y minerales (1 hora)

2. Procesos magmáticos y rocas magmáticas (2 horas y media)

3. Procesos sedimentarios y rocas sedimentarias (2 horas y media)

4. Procesos metamórficos y rocas metamórficas (2 horas)

5. Identificación de rocas y procesos petrogenéticos en el entorno urbano, y realización de un informe final (6 horas)

Los primeros cuatro apartados se realizaron en el aula y en el laboratorio y se centraron en la comprensión de los procesos petrogenéticos y en la identificación y clasificación de muestras. En concreto se pidió al alumnado rellenar varias fichas de identificación de diferentes muestras (ver Figura 1), guiándoles en las características en las que debían fijarse en cada caso. Este ejercicio resultó muy útil como preparación previa a las actividades de la salida urbana, y para cada tipo de roca se dedicaron al menos 30 minutos; además, se les solicitó entregarlo para su evaluación y posterior corrección.

FICHA DE IDETIFICACIÓN DE MUESTRAS DE ROCAS

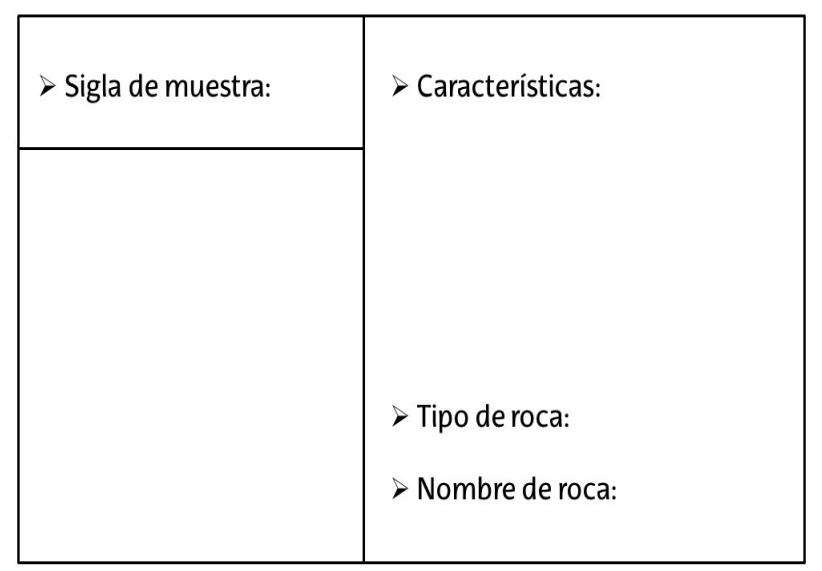

Figura 1. Ficha de identificación a completar por los estudiantes con las muestras, indicando, a la izquierda, la sigla de la muestra y un dibujo de la misma, y a la derecha sus características (tamaño de grano, textura, contenido fósil, foliación, etc.), tipo de roca (ígnea volcánica, ígnea plutónica, sedimentaria detrítica, sedimentaria química, metamórfica de contacto o metamórfica regional) y por último el nombre de la roca.

El quinto apartado corresponde a las actividades relacionadas con la salida (anteriores, durante y posteriores a ella) y es el que se va a desarrollar más específicamente en este artículo. En el momento en el que los grupos comienzan con este apartado, ya han trabajado los procesos por los que se han creado los diferentes tipos de rocas y por tanto las características que 
presentan. Sin embargo, todavía no han aplicado lo aprendido en contextos diferentes, ni han relacionado los diferentes apartados entre sí para dar respuesta a un problema real. Es por ello que la salida urbana no es solamente una actividad complementaria, sino que constituye una actividad de aplicación y síntesis muy relevante en el proceso de enseñanza-aprendizaje (Del Carmen y Pedrinaci 1997).

\section{Actividades y puesta en práctica}

A continuación detallamos las actividades del $5^{\circ}$ apartado, que se dividieron en:

1. Actividades pre-salida, en las que se familiarizó a los estudiantes con el entorno a visitar y se les planteó el problema a resolver: reconocer algunas de las rocas más significativas de su entorno urbano. Durante esta fase tenían que realizar, en grupos, una guía de observación para utilizar durante la salida, que recogiera las hipótesis que tendrían que comprobar. En este caso, como el objetivo era el análisis y reconocimiento de cuatro rocas ornamentales, se les pidió como guía una clave dicotómica.

2. Actividades en la salida, en las que los grupos tenían que hacer uso de su guía (clave dicotómica) y de diferentes recursos (ácido clorhídrico, reglas, cámara de fotos, etc.) para recoger datos y poder concluir la litología de las muestras.

3. Actividades post-salida, empleadas para organizar la información recogida, resolver las dudas surgidas y sintetizar toda la información en un informe científico. En este informe deberían incluir la localización y objetivos del trabajo, la descripción detallada de las rocas analizadas en la salida así como su identificación, y algunas conclusiones.

\section{Actividades pre-salida}

Las actividades anteriores a la salida tienen como objetivo que el alumnado conozca y prepare el problema a resolver en el entorno urbano (análisis e identificación de cuatro rocas ornamentales localizadas en las calles de Orduña). Para ello se les pidió sintetizar la información trabajada en los primeros apartados de la unidad didáctica en una clave dicotómica.

La realización de una clave dicotómica es una actividad muy interesante para organizar las características de las rocas que se han trabajado previamente, como se ha visto en otros trabajos (De la Torre 1992, Vilches, Legarrade y Berasain 2012). Más aún, su correcta realización requiere una profunda comprensión de los procesos formadores de rocas y de las características principales de cada una de ellas, así como relacionar y comparar los tipos de rocas entre sí. De hecho, en ella se clasifican las muestras usando las características representativas de cada roca como criterio (texturas, tamaño de grano, contenido fósil, etc.). Un posible modelo de clave dicotómica para este caso se muestra en la Figura 2, pero cabe recordar que no existe una única forma de realizarlas, y que puede haber otras correctas.

Este ejercicio puede resultar complejo para el alumnado si no se limita el número de rocas posibles. Por ello se explicó a la clase cuáles eran las litologías susceptibles de aparecer en la salida, dentro de cada tipo de roca:

- En las rocas ígneas: granito, gabro, basalto, riolita, pórfido granítico y pegmatita granítica.

- En las rocas sedimentarias: conglomerado, arenisca, lutita, caliza, yeso y halita.

- En las rocas metamórficas: pizarra, esquisto, gneis, mármol y cuarcita.

Los estudiantes trabajaron en grupos en el laboratorio durante una hora, sintetizando la información trabajada en el aula previamente (en especial hicieron uso de las fichas de identificación que habían realizado y de todas las muestras de rocas que tenían a su 
disposición). Surgió algún problema al comenzar con la clave, pero una vez entendido el mecanismo, consiguieron avanzar. A pesar de ello el tiempo previsto no fue suficiente y tuvieron que terminar el trabajo fuera de la hora de clase.

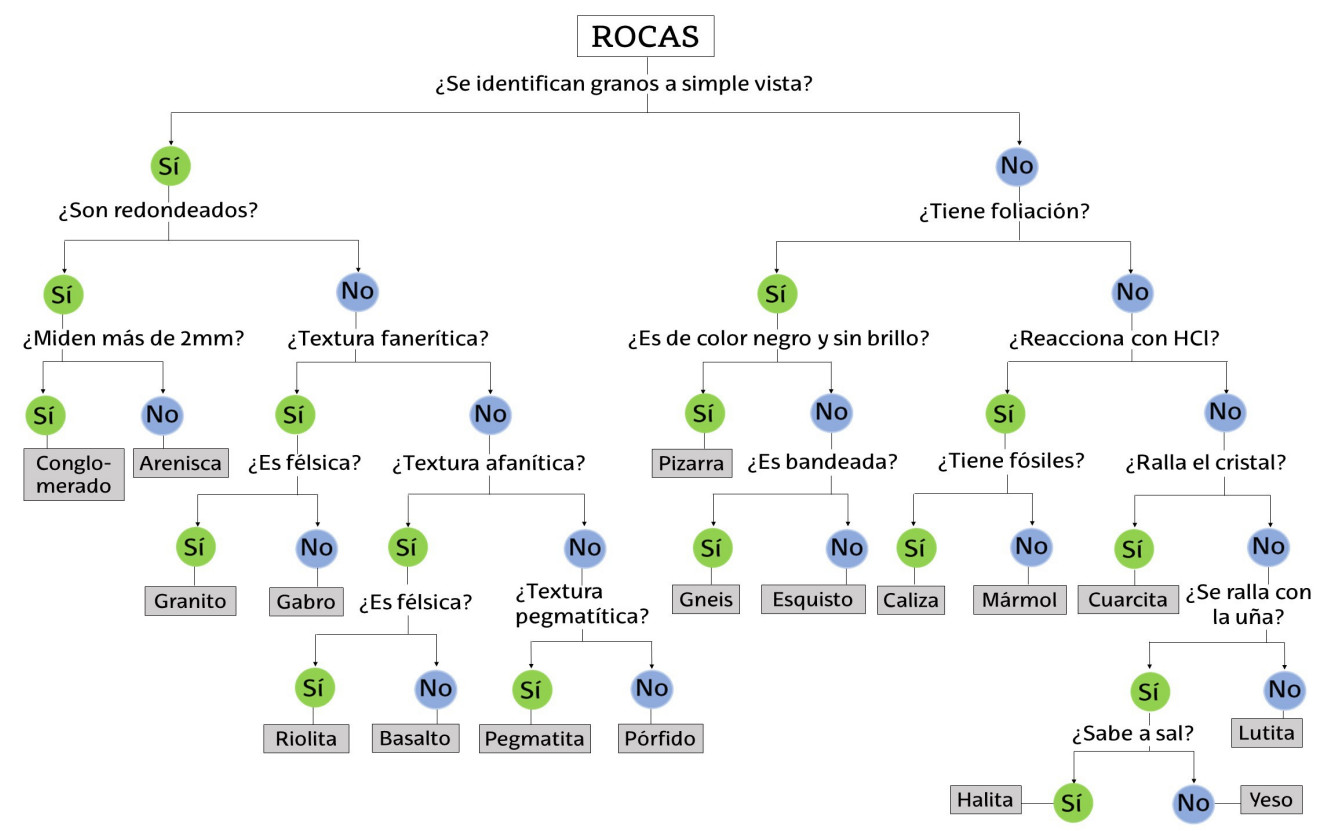

Figura 2. Modelo de clave dicotómica.

Esta actividad no sólo fue interesante por sí sola como ejercicio de síntesis, sino que además resultó ser adecuada para introducir la salida urbana. Gracias a ella, los chicos y chicas pudieron reflexionar sobre lo que esperaban encontrar en la salida, y por tanto, prever las dificultades y prepararse para enfrentarlas.

\section{Actividades en la salida}

La duración de la salida fue de dos horas, y se realizó a través de las calles de Orduña (calle Burgos y alrededores de la parroquia de Santa María, concretamente). Se trabajó con cuatro muestras, siendo la distancia entre ellas de 200-300 metros aproximadamente.

El quehacer en cada parada se había dividido en dos partes. En primer lugar, se les pedía un ejercicio de orientación: a cada grupo se le había entregado un mapa aéreo de la zona, para indicar en él la localización de la muestra. Después debían comenzar con el análisis e identificación, haciendo uso de la clave dicotómica y de los recursos que se habían acordado en clase.

Las rocas escogidas para esta salida eran muestras representativas de cada tipo (ígneas, sedimentarias y metamórficas), que tenían un corte limpio (bien pulido) y no estaban meteorizadas. En concreto, se trabajaron las siguientes (ver Figura 3):

1. Muestra: Mármol bandeado (fachada)

2. Muestra: Caliza Negro Markina, contiene fósiles de rudistas (adoquinado)

3. Muestra: Granito rosa (fachada)

4. Muestra: Caliza Rojo Ereño, contiene fósiles de rudistas (adoquinado) 


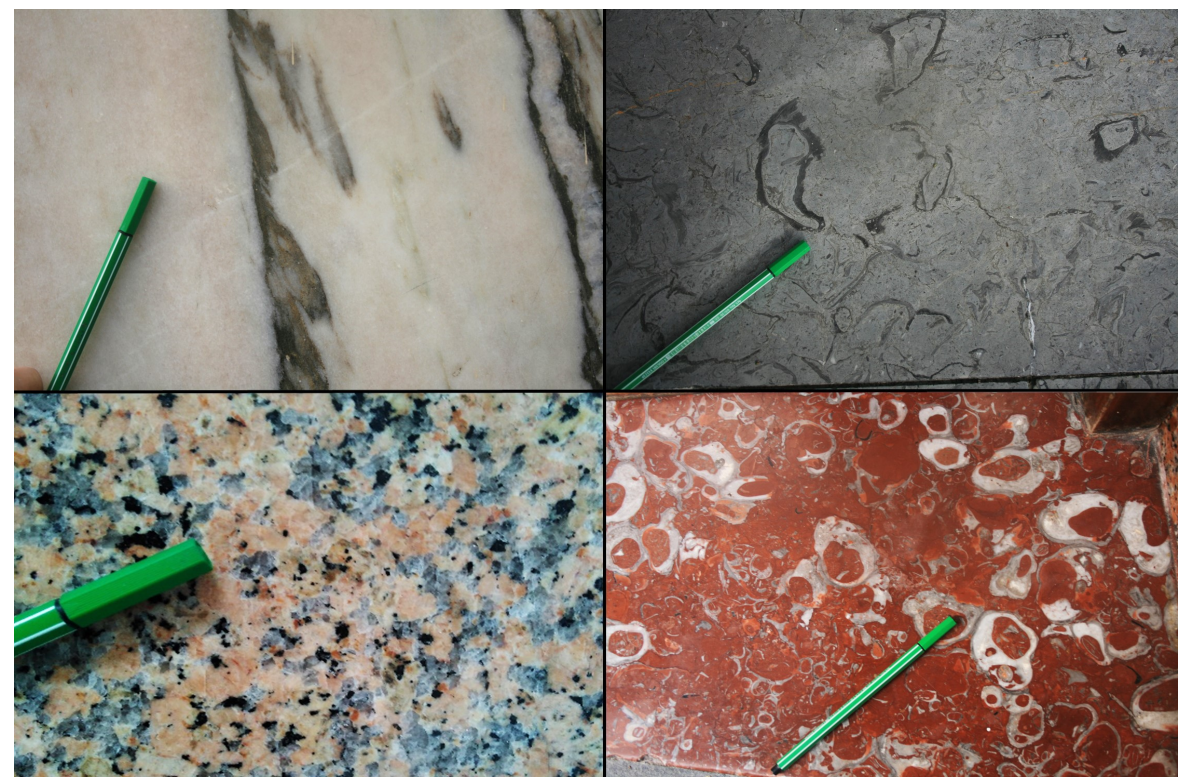

Figura 3. Imágenes de las rocas analizadas por los estudiantes. En la primera línea, de izquierda a derecha: mármol bandeado y caliza Negro Markina. En la segunda línea, de izquierda a derecha: granito rosa y caliza Rojo Ereño.

El motivo por el que se decidió trabajar con sólo cuatro rocas fue la duración prevista para el análisis de cada una, y la duración de la salida en sí. Debido a que no estaban acostumbrados a trabajar de forma autónoma y menos en la descripción e identificación de rocas, se optó por no cargar el trabajo de los grupos con más muestras y dar preferencia a la calidad frente a la cantidad de los análisis. Por otro lado, cabe destacar que dos de las muestras que se eligieron (las calizas Negro Markina y Rojo Ereño) forman parte del patrimonio geológico del País Vasco (Damas-Mollá et al. 2012), siendo litotipos internacionalmente conocidos y muy habituales en el entorno de estos estudiantes.

Durante el desarrollo de la salida el ejercicio de orientación y el análisis de las muestras fueron ejecutados sin problemas y en el tiempo establecido. El alumnado conocía perfectamente el objetivo y trabajaba de manera bastante autónoma, si bien es cierto que en ocasiones, algunos estudiantes acudían a la profesora ante la más mínima duda; en estos casos, se intentó no interferir y animarles a dialogar entre sí para resolver los problemas.

\section{Actividades post-salida}

Después de la salida se les propuso elaborar un informe científico, organizando y sintetizando la información recogida, y para ello se emplearon tres horas.

Inicialmente trabajaron en grupos, en una sala de ordenadores. Este tiempo sirvió para que el alumnado pudiese solucionar dudas y para que las docentes pudiesen identificar algunas dificultades. Así se observó que este ejercicio les resultó más difícil de lo esperado: aunque tenían la información, no sabían cómo enfocar el informe, ni tampoco emplear correctamente el lenguaje científico. La mayoría relataba lo sucedido en la salida utilizando la primera persona, y no explicaban, en general, las pruebas que les habían llevado a la identificación de las muestras. Tuvieron también problemas para redactar las conclusiones.

A la vista de estos obstáculos y aunque no estaba previsto, se proporcionaron unas pautas sobre cómo elaborar un informe científico (cuáles eran los apartados y qué se pedía en cada uno), y se resolvieron las dudas sobre la redacción del mismo. El funcionamiento de los grupos difirió respecto a las actividades realizadas previamente: en este caso se apreció que 
algunos estudiantes asumían más carga de trabajo que otros. Además, el tiempo previsto no fue suficiente y -a excepción de un grupo- tuvieron que terminar el informe en casa.

Por último, en la última hora se realizó la evaluación final. Los grupos entregaron el informe solicitado (que las profesoras calificaron mediante una rúbrica) y se evaluó y calificó el trabajo cooperativo (utilizando para ello una rúbrica de coevaluación del trabajo en grupo). Después se evaluó la intervención en general, mediante un post-test anónimo sobre contenidos en el que también se solicitaba la opinión del alumnado.

\section{Herramientas de evaluación}

Tras la concreción de los objetivos y el diseño de las actividades se analizaron las posibilidades de valorar el trabajo realizado, seleccionando varias herramientas de evaluación (que complementan la observación in-situ): un post-test a realizar por los estudiantes al final de la secuencia, una rúbrica de evaluación para el informe grupal final y, otra rúbrica para realizar la coevaluación entre los integrantes de un mismo grupo (ver rúbricas en los Apéndices I y II). Los detalles se muestran en la tabla I:

Tabla I. Aspectos evaluados en la intervención y herramientas empleadas para ello.

\begin{tabular}{|l|l|}
\hline ASPECTOS EVALUADOS & HERRAMIENTAS \\
\hline \multirow{2}{*}{ Asimilación de contenidos (conceptos y procedimientos) } & Informe científico grupal (corregido con Rúbrica) \\
\cline { 2 - 2 } & Post-test (anónimo) \\
\hline \multirow{2}{*}{ Asimilación de contenidos (actitudes) } & Coevaluación entre alumnos/as (con Rúbrica) \\
\cline { 2 - 2 } & Post-test (anónimo) \\
\hline
\end{tabular}

Tanto las dos rúbricas empleadas como las preguntas del test fueron consensuadas entre las autoras y la profesora del centro. Así mismo, el análisis de los resultados se realizó de forma conjunta entre estas tres personas. Aunque el grupo es pequeño y los resultados no pueden ser generalizables, en esta investigación se ha utilizado una metodología mixta (cualitativa y cuantitativa) para obtener unas conclusiones fundamentadas.

\section{Valoración de la secuencia}

\section{Mediante observación}

En relación a las dificultades observadas en las actividades previas a la salida, destaca que el tiempo previsto para la realización de la clave dicotómica no fue suficiente. Sin embargo, esta actividad fue, probablemente, en la que más destacó la implicación del alumnado. Tanto fue así que la mitad de la clase decidió quedarse en el laboratorio en la hora del recreo a fin de terminar dicha clave. Además, el funcionamiento de los grupos fue bastante equilibrado: en general participaban todos, conversaban sobre cómo seguir, se repartían el trabajo y se ayudaban mutuamente.

Las actividades realizadas durante el recorrido por las calles de Orduña se hicieron sin problemas. Dado que el grupo era pequeño y había dos profesoras durante la salida, se pudo realizar la observación adecuadamente. Se vio que todos los grupos analizaron las rocas con mucho interés, sacando numerosas fotos para adjuntar en el informe y, también destacó la alta participación y la colaboración dentro de cada grupo. A pesar de que solo se les había pedido que trabajaran con cuatro muestras, algunos estudiantes se dieron cuenta de que su ciudad 
estaba repleta de una amplia variedad de rocas; hicieron muchas preguntas sobre ellas, y mostraron curiosidad y fascinación por el redescubrimiento de las conocidas calles.

Por último, y respecto a las actividades finales, se apreció que los grupos trabajaron con más dificultad y no tan entusiasmados como al principio. Quizá por la falta de costumbre en la realización de informes científicos, no sabían enfocar bien el trabajo. Es representativo el hecho de que, aunque se les propuso entregar un borrador para que fuese evaluado antes de la calificación y así tuviesen tiempo de corregirlo, ningún grupo lo presentó. No se comentó este aspecto con el alumnado, pero creemos que la razón fue el escaso tiempo que tenían para la realización de esta actividad.

\section{Mediante herramientas de evaluación}

\section{Informes cientificos grupales}

Tras valorar mediante la rúbrica el informe entregado por cada grupo, se obtuvieron buenos resultados: todos los informes obtuvieron buena calificación, con una nota media de 4,59 sobre 6. La nota más alta fue 5,38 sobre 6, y la nota más baja 3,69.

Entre todos los aspectos evaluados (ver rúbrica en el Apéndice I), los que obtuvieron mejor puntuación fueron la localización e identificación de las rocas en la calle, y las claves dicotómicas, algo que ya se preveía de antemano porque estas claves les resultaron muy útiles en la salida. En cambio, la principal dificultad detectada fue la inadecuada utilización del lenguaje. En menor medida se observaron problemas con la descripción de las muestras ya que sólo un grupo omitió algún elemento importante para la identificación de las rocas. Estos fallos evidencian la falta de costumbre y preparación para realizar este tipo de trabajos.

\section{Post-test}

Los resultados de la primera parte del post-test (realizado individualmente) se han valorado de forma positiva: 6 estudiantes respondieron correctamente a más de la mitad de las preguntas, siendo la nota media de la clase de 5,2 sobre 10. Dado que los estudiantes sabían que las respuestas no iban a influir en su calificación, pensamos que alguno de ellos no se implicó demasiado a la hora de responder.

Todas las preguntas eran de tipo test, con una única opción correcta a elegir entre tres, a excepción de la segunda cuestión, en la que se les pedía un dibujo sobre la génesis de cualquier roca. El criterio para corregir esta pregunta fue que se mostrase con claridad en el dibujo, como mínimo, un proceso petrogenético. Las preguntas del test y el número de aciertos y fallos en cada una se muestran en la tabla II.

Los resultados de este cuestionario evidencian la minimización de algunos errores conceptuales habituales en geología, como la naturaleza de las rocas y los minerales (preguntas 1 y 4), o el dinamismo de la Tierra (preguntas 11 y 12). También se observa que el alumnado conoce los criterios de clasificación de las rocas detríticas (pregunta 13) y que recuerda la forma de diferenciar el mármol de otras rocas no carbonatadas (pregunta 17). Sin embargo, los aspectos relacionados con los procesos petrogenéticos que ocurren en profundidad (preguntas 3 y 15) y los criterios de clasificación de las rocas ígneas (preguntas 5, 6, 7, 8 y 9) no han quedado claros. Los dibujos realizados en la segunda pregunta, que fueron en su mayoría volcanes en erupción y/o procesos detríticos, evidencian también las dificultades con los procesos petrogenéticos internos.

Estos resultados probablemente se deban a dos razones: a) la dificultad que entraña imaginar y entender procesos geológicos internos como el magmatismo y el metamorfismo, ya que son invisibles y ocurren a escalas espacio-temporales desconocidas para el alumnado de esta edad; 
b) el tipo de preguntas formuladas: el haber preguntado de manera teórica requería recordar muchos tecnicismos con los que los estudiantes no se habían familiarizado (como "magma evolucionado", "textura afanítica", "composición máfica", etc.), lo que dificultó responder correctamente sin haber memorizado.

Tabla II. Primera parte del post-test realizado de manera anónima en clase al terminar la UD. En él se evalúan aspectos conceptuales y procedimentales del temario. (Las respuestas correctas se han resaltado en negrita)

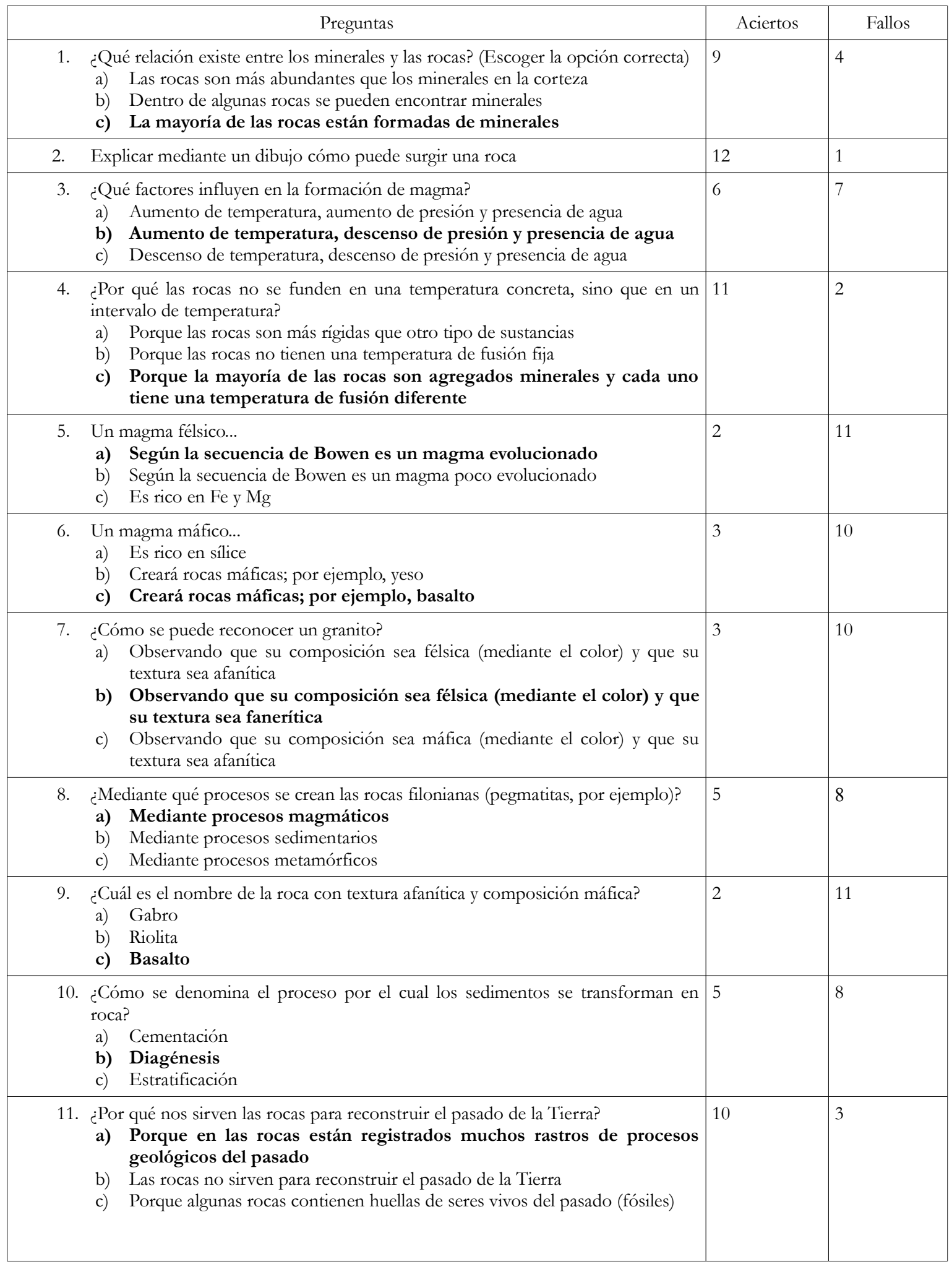


Tabla II. Continuación

\begin{tabular}{|c|c|c|}
\hline Preguntas & Aciertos & Fallos \\
\hline $\begin{array}{l}\text { 12. Si encontramos algunos estratos en posición vertical, ¿qué podemos pensar? } \\
\text { a) Que se depositaron en vertical } \\
\text { b) Que se depositaron en horizontal y que después se deformaron } \\
\text { c) Que no están en su posición natural, porque las habrá movido el ser } \\
\text { humano }\end{array}$ & 9 & 4 \\
\hline $\begin{array}{l}\text { 13. ¿Cómo se diferencian las rocas detríticas entre ellas? } \\
\text { a) Por la textura } \\
\text { b) Por el tamaño de grano } \\
\text { c) Por su estructura }\end{array}$ & 12 & 1 \\
\hline $\begin{array}{l}\text { 14. Las calizas... } \\
\text { a) Son rocas sedimentarias orgánicas } \\
\text { b) Están formadas de minerales de calcita, que tienen esta composición: } \\
\text { CaCO3 } \\
\text { c) No son abundantes en Orduña }\end{array}$ & 4 & 9 \\
\hline $\begin{array}{l}\text { 15. Cuando ocurre el metamorfismo regional... } \\
\text { a) Ocurre la recristalización de los minerales } \\
\text { b) Los minerales se ordenan siguiendo la orientación preferente } \\
\text { c) Ocurre metamorfismo de especialmente muy altas temperaturas }\end{array}$ & 6 & 7 \\
\hline $\begin{array}{l}\text { 16. La roca cuarcita... } \\
\text { a) Surge mediante metamorfismo regional } \\
\text { b) Surge del metamorfismo de una arenisca } \\
\text { c) Es de color negro }\end{array}$ & 7 & 6 \\
\hline $\begin{array}{l}\text { 17. ¿Cómo se puede diferenciar un mármol de una cuarcita? } \\
\text { a) El mármol se forma mediante metamorfismo regional y la cuarcita mediante } \\
\text { metamorfismo de contacto } \\
\text { b) El mármol tiene composición carbonatada y la cuarcita no } \\
\text { c) El mármol tiene brillo y la cuarcita no }\end{array}$ & 9 & 4 \\
\hline
\end{tabular}

\section{Coevaluación}

Analizando las rúbricas de evaluación del trabajo en grupo (en las que los y las estudiantes se evaluaron entre sî), se observa que el funcionamiento de casi todos los grupos fue bueno: en tres grupos la puntuación fue de 1,6-2 sobre 2 y sólo en un grupo se obtuvieron puntuaciones inferiores, de 0,83-1,5 sobre 2. Por tanto, podemos decir que tres de los cuatro grupos trabajaron bien y de forma cooperativa. Es reseñable que los participantes del grupo restante completasen las rúbricas con coherencia y honradez, a pesar de tener que calificar negativamente a algún compañero.

\section{Motivación e interés por la geología}

Por último, son de gran interés los resultados de la segunda parte del post-test, en la que se pidió al alumnado su opinión sobre la salida urbana (ver Tabla III). La mayoría dice estar de acuerdo con la forma de hacer la salida - autónoma mediante un problema que investigar-, e indica que la realización de la clave dicotómica fue útil para ello. Se muestran asimismo conformes con la utilidad del informe para afianzar conceptos, a pesar de las dificultades que tuvieron en este proceso. Además, todo el grupo indica que la actividad fue motivadora, y el interés por la geología se puede considerar aceptable (media de 3,8 sobre 5). 
Tabla III. Valoración del 1 al 5 (1-Muy en desacuerdo, 5-Muy de acuerdo) por parte de los estudiantes de la metodología empleada (Segunda parte del post-test).

\begin{tabular}{|c|c|c|c|c|c|}
\hline Preguntas & 1 & 2 & 3 & 4 & 5 \\
\hline & \multicolumn{5}{|c|}{ Número de estudiantes } \\
\hline $\begin{array}{l}\text { 20. La elaboración del informe después de la salida me } \\
\text { ha ayudado a entender los conceptos que se habían } \\
\text { quedado sin resolver. }\end{array}$ & 0 & 0 & 0 & 8 & 5 \\
\hline 23. Tengo un alto interés por la geología & 0 & 1 & 5 & 6 & 1 \\
\hline
\end{tabular}

\section{Conclusiones}

La valoración realizada por las profesoras sobre el desarrollo general de la intervención es positiva, ya que los chicos y chicas trabajaron de manera independiente en sus grupos de trabajo y también cooperando entre sí, en un entorno de trabajo relajado.

Sin embargo, durante el proceso se encontraron algunas dificultades o puntos débiles: por un lado que el tiempo previsto para realizar la clave dicotómica fue escaso, y, por otro, que el alumnado no conocía las pautas para realizar el informe científico. Para subsanar estos problemas proponemos flexibilizar la programación y adaptarla al nivel de la clase, tal y como se hizo en la implementación.

Del análisis de los resultados de las rúbricas y el test se extraen tres conclusiones principales: La primera, que el estudiantado ha aprendido la mayoría de los contenidos que se querían trabajar en este tema, y así han mejorado en el desarrollo de la competencia científica. Como se ha indicado previamente todos los informes y las dinámicas de grupo fueron valoradas positivamente, y se trabajaron aspectos como la identificación de variables, la interpretación crítica de resultados y la comunicación de estos, la elaboración de informes y la exactitud en la utilización del lenguaje científico... aspectos que contribuyen a la adquisición de la competencia científica. Si bien en el post-test la nota media de la clase no fue elevada (5'2 sobre 10), pensamos que comprender los procesos petrogenéticos internos y ser capaz de utilizar la terminología adecuada para definir las texturas y composición de las rocas magmáticas, es un proceso que requiere más tiempo del empleado en esta experiencia. Tal y como se concluye en otros estudios, el aprendizaje de aspectos competenciales conlleva siempre una dificultad mayor que el de contenidos específicos (Benarroch y Núñez 2015). Así, la propuesta de mejora en este caso sería dedicar más tiempo al trabajo de laboratorio previo (análisis e identificación de muestras) y adecuar las preguntas del post-test.

En segundo lugar, se concluye que el alumnado ha valorado la metodología como útil, incluida la realización del informe que fue el apartado más problemático de la experiencia.

Por último, y como tercera conclusión, el grupo considera la salida de campo como una actividad motivadora y además, la mayoría indica tener un interés medio-alto por la geología, lo que nos indica que la secuencia didáctica ha sido adecuada. 
Este trabajo coincide con los resultados obtenidos por otros estudios en cuanto a que salir al campo es positivo para aprender geología, pero que para asegurar el aprendizaje significativo es necesario superar ciertos obstáculos mediante una planificación estricta. La experiencia desarrollada ha pretendido proponer una salida urbana realizable y útil, que permita contextualizar la geología y desarrollar la competencia científica sin necesidad de grandes recursos ni desplazamientos. De esta forma, junto con otras experiencias realizadas y por realizar, se espera optimizar la planificación de las salidas de campo y, así, ampliar su presencia en el sistema educativo.

\section{Agradecimientos}

Nos gustaría agradecer a Beatriz Martínez Rodríguez, profesora de Biología y Geología de Educación Secundaria, así como al Colegio Ntra. Sra. de la Antigua de Orduña, por hacer posible el desarrollo de esta intervención y por su actitud abierta y colaboración constante en todo el proceso. Así mismo, queremos agradecer la participación y la inmejorable respuesta que los estudiantes de este grupo de $1^{\circ}$ de Bachillerato de dicho colegio han demostrado durante todo el proyecto.

\section{Referencias}

Benarroch A. y Núñez G. (2015) Aprendizaje de competencias científicas versus aprendizaje de contenidos específicos. Una propuesta de evaluación. Enseñanza de las Ciencias 33 (2), 927.

Branda L.A. (2009) El aprendizaje basado en problemas. De herejía artificial a res popularis. Educación Médica 12 (1), 11-23.

Brusi D., Zamorano M., Casellas R.M., Bach J. (2011) Reflexiones sobre el diseño por competencias en el trabajo de campo en Geología. Enseñanza de las Ciencias de la Tierra 19 (1), 4-14.

Cañal P. (2012) El desarrollo de la competencia científica demanda y produce actitudes positivas hacia la ciencia y el conocimiento científico. En Pedrinaci E. (coord.), Caamaño A., Cañal P. y De Pro A. El desarrollo de la competencia cientifica. 11 ideas clave. Barcelona. Graó.

Cañas A, Martín-Díaz M.J. (2010) ¿Puede la competencia científica acercar la ciencia a los intereses del alumnado? Alambique 66, 80-87.

Corbí H., Giannetti A., Baeza-Carratalá J.F. y Martínez-Martínez J. (2013) Elaboración de itinerarios geológicos como recurso didáctico en Ciencias de la Tierra. Actas de las XI Jornadas de Redes de Investigación en Docencia Universitaria. Universidad de Alicante. Alicante.

Decreto 127/2016 de 6 de septiembre, por el que se establece el currículo de Bachillerato y se implanta en la Comunidad Autónoma del País Vasco. (BOPV 23-09-16)

De la Torre E. (1992) La utilización de los diagramas de flujo para la identificación de las rocas en el campo. Investigación en la escuela 16, 101-102.

Del Carmen L. y Pedrinaci E. (1997) El uso del entorno y el trabajo de campo. En Del Carmen, L. (coord.): La enseñanza y el aprendizaje de las ciencias de la naturaleza en la educación secundaria (Cap V). Barcelona: Horsori.

Del Toro R. y Morcillo J.G. (2011) Las actividades de campo en educación secundaria. Un estudio comparativo entre Dinamarca y España. Enseñanza de las Ciencias de la Tierra 19 (1), 39-47. 
Damas-Mollá L., Aranburu A., Garcia-Garmilla P. y Fano H. (2012) Rocas ornamentales del País Vasco y Navarra (I): El Rojo Ereño y el Negro Markina. Tierra y Tecnología 42, 25-33.

Fuertes I., De la Calzada E., Llamas T., Tejerina A., Crespo M.A., Pereiras L., Crespo T., Domínguez L. y Cabezas L. (2016) Lugares de interés geoeducativo en el medio urbano. Potencialidad de las ciudades para la enseñanza de la geología. Enseñanza de las Ciencias de la Tierra 24 (2) 195-201.

Morentin M. y Guisasola J. (2013) Visitas escolares a centros de ciencias basadas en el aprendizaje. Alambique 73, 61-68.

OECD (2016) PISA 2015 Assessment and Analytical Framework: Science, Reading, Mathematics and Financial Literacy. París: PISA, OECD Publishing.

Pavón F. y Martínez Aznar Mª M. (2014) La metodología de resolución de problemas como investigación (MRPI): una propuesta indagativa para desarrollar la competencia científica en alumnos que cursan un programa de diversificación. Enseñanza de las Ciencias 32 (3), 469-492.

Pedrinaci E. (1996) Sobre la persistencia o no de las ideas del alumnado en geología. Alambique 7, 27-36.

Pedrinaci E. (2012a) Alfabetización en Ciencias de la Tierra, una propuesta necesaria. Enseñanæa de las Ciencias de la Tierra 20 (2), 133-140.

Pedrinaci E. (2012b) Trabajo de campo y aprendizaje de las ciencias. Alambique 71, 81-89.

Pedrinaci E., Ruiz de Almodóvar G., Alfaro P., Brusi D., Fernández E., Pascual J.A., Alcalde S., Barrera J.L., Belmonte A., Calonge A., Cardona V., Crespo A., Feixas J.C., González-Díez A., Jiménez J., Mata J.M., López -Ruiz J., Quintanilla L., Rábano I., Rebollo L., Rodrigo A. y Roquero E. (2012) Alfabetización en ciencias de la Tierra. Actas del XVII Simposio sobre Enseñanza de la Geología (pp: 296-309) Universidad de Huelva. Huelva.

Pedrinaci E., Sequeiros L. y García de la Torre E. (1994) El trabajo de campo y el aprendizaje de la Geología. Alambique 2, 37-45.

Ramos R., Praia J., Marqués L. y Pereira L.G. (2001) Ideas alternativas sobre el ciclo litológico en alumnos portugueses de enseñanza secundaria. Enseñanza de las Ciencias de la Tierra 9 (3), 252-260.

Ryan R. M. y Deci E. L. (2000) The 'what' and 'why' of goal pursuits: Human needs and the self-determination of behavior. Psychological Inquiry 11, 227-268.

Sanmartí N. (2007) 10 ideas clave: Evaluar para aprender. Barcelona: Graó.

Sanmartí N. y Márquez C. (2017) Aprendizaje de las ciencias basado en proyectos: del contexto a la acción. Apice. Revista de educación científica 1 (1), 3-16.

Vilaseca A. y Bach J. (1993) ¿Podemos evaluar el trabajo de campo? Enseñanza de las Ciencias de la Tierra 1 (3), 158-167.

Vilches A., Legarralde T. y Berasain G. (2012) Elaboración y uso de claves dicotómicas en las clases de biología. Actas de las III Jornadas de Enseñanza e Investigación Educativa en el campo de las Ciencias Exactas y Naturales. Facultad de Humanidades y Ciencias de la Educación. Universidad Nacional de La Plata, Buenos Aires, Argentina.

Zamalloa T., Maguregi G., Fernández D., Echevarría I. y Sanz J. (2014) Acercar la geodiversidad a través de las salidas de campo en la ESO. Una investigación con el profesorado de ciencias de Bizkaia. Enseñanza de las Ciencias 32 (3), 443-467. 